Review Article

\title{
Effects of Physical Exercises on Pulmonary Rehabilitation, Exercise Capacity, and Quality of Life in Children with Asthma: A Meta-Analysis
}

\author{
YiRan Liu $\mathbb{D}^{1},{ }^{1}$ Yan Zhao $\mathbb{D}^{1},{ }^{1}$ Fang Liu $\mathbb{D}{ }^{2}$ and Lin Liu $\mathbb{D}^{1}$ \\ ${ }^{1}$ School of Sport and Health, Nanjing Sport Institute, Nanjing 210014, Jiangsu, China \\ ${ }^{2}$ Respiratory Medicine of Xuyi People's Hospital, Huaian 223001, Jiangsu, China \\ Correspondence should be addressed to Lin Liu; liulinst1989@126.com
}

Received 10 August 2021; Revised 3 December 2021; Accepted 6 December 2021; Published 23 December 2021

Academic Editor: Sabina Barrios-Ferrandez

Copyright (c) 2021 YiRan Liu et al. This is an open access article distributed under the Creative Commons Attribution License, which permits unrestricted use, distribution, and reproduction in any medium, provided the original work is properly cited.

Objective. This study aimed to systematically evaluate the effect of exercise on pulmonary function, exercise capacity, and quality of life in children with bronchial asthma. Methods. A comprehensive search was performed using PubMed, Cochrane Library, Web of Science, EBSCO, CNKI, and Wanfang Data Knowledge Service platform to identify any relevant randomized controlled trials (RCTs) published from inception to April 2021. The Cochrane risk of the bias tool was utilized to evaluate the methodological quality of the included studies, and RevMan 5.3 was applied to perform data analyses. Results. A total of 22 RCTs involving 1346 patients were included. The results of the meta-analysis showed that exercise had significant advantages in improving lung function and exercising capacity and quality of life in children with asthma compared with conventional treatment, such as the forced vital capacity to predicted value ratio ( $\mathrm{SMD}=0.27 ; 95 \% \mathrm{CI}: 0.13,0.40$, and $P<0.0001$ ), the peak expiratory flow to predicted value ratio $(\mathrm{MD}=4.53 ; 95 \% \mathrm{CI}: 1.27,7.80$, and $P=0.007)$, the 6 -minute walk test $(\mathrm{MD}=110.65 ; 95 \% \mathrm{CI}$ : $31.95,189.34$, and $P=0.006)$, rating of perceived effort (MD $=-2.28$; $95 \% \mathrm{CI}:-3.21,-1.36$, and $P<0.0001)$, and peak power $(\mathrm{MD}=0.94 ; 95 \% \mathrm{CI}$ : $0.37,1.52$, and $P=0.001)$ on exercise capacity and pediatric asthma quality of life questionnaire $(\mathrm{MD}=1.28 ; 95 \% \mathrm{CI}: 0.60,1.95$, and $P=0.0002$ ) on quality of life. However, no significant difference was observed in the forced expiratory flow between $25 \%$ and $75 \%$ of vital capacity $(P=0.25)$ and the forced expiratory volume at 1 second to predicted value ratio $(P=0.07)$. Conclusions. Current evidence shows that exercise has a certain effect on improving pulmonary function recovery, exercise capacity, and quality of life in children with bronchial asthma. Given the limitation of the number and quality of included studies, further research and verification are needed to guide clinical application.

\section{Introduction}

Bronchial asthma is a common chronic respiratory disease in children, and the etiology is unclear. Most studies indicate that asthma is related to genetic and environmental factors, such as allergic constitution, viral infection, particulate matter, smoke, and ozone exposure [1]. The main clinical manifestations of asthma include breathlessness, cough, shortness of breath, and wheezing, which are easy to attack or aggravate at night and in the early morning [2] and need long-term treatment because of repeated attacks and prolonged treatment, which may lead to irreversible damage to lung function. In severe cases, these manifestations will affect the growth, development, exercise ability, and quality of life of children. The number of patients with global bronchial asthma increases annually with the rapid pace of modern society and the progress of science and technology, particularly in children [3]. According to the statistics, at present, more than 300 million children suffer from bronchial asthma worldwide, and global analysis of the ISAAC study and other reports showed that the prevalence of asthma averaged approximately $10 \%$ for 6-7 years old [4]. Therefore, effectively preventing and treating childhood asthma has become a focus of global research.

At present, the prevention and treatment of asthma are primarily based on drugs, such as glucocorticoids, 
antihistamines, $\beta-2$ agonists, and leukotriene receptor antagonists [5]. However, the effect of most drugs is limited, and they will have several side effects; therefore, finding a safe and effective alternative therapy has become the focus of pulmonary physicians. In recent years, considerable attention has been paid to the role of exercise in alleviating airway inflammation and delaying or reversing airway remodeling. During the outbreak of novel coronavirus pneumonia (COVID-19) in China, exercise, as an important means for the recovery of pulmonary function of COVID-19 patients, is highly recommended by medical staff during the development of the epidemic. It is also included in COVID-19's rehabilitation guidelines for integrated traditional Chinese and Western medicine (first edition) [6]. Based on the Global Initiative for Asthma (GINA) guidelines, children with asthma should participate in physical exercises like normal children, which is considered to be an important part of the nondrug prevention and treatment strategy for children with asthma. Therefore, exercise has also been used in the rehabilitation of lung function, exercise ability, and quality of life in children with bronchial asthma, but the prevention and treatment effects are different [7-12]. Andrade et al. [11] conducted a 6-week randomized controlled trial (RCT) on 33 children with moderate asthma. The study showed that aerobic exercise intervention had significant advantages over nonexercise in improving asthma-related symptoms, lung function, exercise ability, and quality of life. However, exercise was once regarded as the inducing factor of asthma, particularly in patients with exercise-induced asthma, and children would reduce or give up exercise because of repeated attacks. Meta-analysis shows that swimming for 6-12 weeks is significantly better than routine treatment in improving pulmonary function indexes such as FEV $1 \%$ pred and FEF25-75\% pred in children with bronchial asthma, but no significant difference was observed in FVC $\%$ pred, FEF50\% pred, and other indexes [12]. A previous systematic review on 11 studies by Zhang et al. [13] found physical training improved FVC (\% pred) significantly in children with asthma. This finding may support the therapy of physical training in asthmatic children. But further research involving the physical training mode, the duration, frequency, and other outcome indicators is needed. Therefore, the extensive application of exercise in children with bronchial asthma is difficult.

To date, whether exercise can be included in the rehabilitation of lung function in children with bronchial asthma and whether exercise can improve children's pulmonary function, exercise ability, and quality of life remains unknown. Therefore, this paper intends to systematically evaluate the intervention effect of exercise on the improvement of lung function, exercise ability, and quality of life in children with bronchial asthma by metaanalysis, thereby objectively and quantitatively evaluating the effectiveness of exercise rehabilitation and providing a scientific and rigorous basis for clinical respiratory and rehabilitation doctors in their clinical work and scientific research.

\section{Materials and Methods}

This systematic review was conducted and reported in accordance with the Preferred Reporting Items for a Systematic Review and Meta-Analysis Framework $[14,15]$. We did not publish or register a protocol for this study.

2.1. Search Strategy. A predetermined search strategy was implemented until April 2021 using the PubMed, Cochrane Library, Web of Science, EBSCO, China Knowledge Network, and Wanfang data knowledge service platform for RCTs on the effect of exercise on children with bronchial asthma. Search strategies included a combination of text words and subject headings (such as MeSH and free terms) relating to (1) physical activity or exercise; (2) asthma; (3) child; and (4) random or allocation. We did not explicitly include search terms related to pulmonary rehabilitation or exercise capacity in the search strategies as these terms were rarely mentioned in the title, abstract, or subject headings, but they were often embedded within full-text articles; thus, they must be identified at the screening level.

2.2. Study Selection. We considered the participant, intervention, comparison, outcome, time, and study (PICOTS) design criteria to ascertain the study inclusion criteria. The inclusion criteria comprised the following: (1) Types of studies: This meta-analysis included only RCTs about the effects of exercise and routine exercise prescriptions on children and adolescents with bronchial asthma. (2) Research subjects: All met the revised standard of the Respiratory Group of the Chinese Medical Pediatrics Association [16] or the diagnostic standard of GINA children with asthma [17]. In addition, the disease was in the nonacute stage, and the sex, race, and nationality of the subjects were not restricted. (3) Intervention: Exercise was based on routine treatment, including aerobic training (swimming, ball games, rope skipping, jogging, and cycling), strength training, and balance and coordination training. (4) Control: Routine treatment includes medication, nutritional support, family breathing exercises, or routine activities without exercise. (5) Outcome: Pulmonary function indices include the forced vital capacity to predicted value ratio (FVC\% pred), the forced expiratory volume at $1 \mathrm{~s}$ to predicted value ratio (FEV1\%pred), the forced expiratory flow between $25 \%$ and $75 \%$ of vital capacity ratio (FEF $25-75 \%$ pred), and the peak expiratory flow to predicted value ratio (PEF\%pred). Exercise capacity indices include the 6-minute walk test (6MWT), rating of perceived effort (RPE), and peak power (PP). Quality of life indicators include Pediatric Asthma Quality of Life Questionnaire (PAQLQ): symptom score, activity score, and emotional score.

The exclusion criteria comprised the following: (1) reviews, guidelines, letters, commentaries, book chapters, or published only as an abstract or poster; (2) studies of incomplete design; (3) full-text literature that is not available through various channels and methods; (4) research articles of poor quality and lack of access to quality information; and (5) the sample size of less than 10 cases. 
2.3. Data Extraction. The two researchers independently screened the literature on the basis of the admission and exclusion criteria, extracted the data, checked each other after the completion of the statistics, and discussed the main points or referred to the opinions of the third party in case of disagreement. Extraction data include first author, year of publication, research methods, sample size, intervention measures, treatment frequency, treatment time, and outcome index. For trials with more than two intervention groups, the experimental group was compared with the control group by combining the data of all relevant control groups [18]. If required information was not reported, then we tried to request it from the corresponding author of the studies and exclude the literature if no other channel information was available or the author had no response [18].

2.4. Quality Assessment. Two reviewers were involved in the risk of bias assessment. The risk of bias was assessed using the Cochrane Collaboration tool, which contained six domains (random sequence generation, allocation concealment, blinding of participants and personnel, blinding of outcome assessors, incomplete outcome data, selective reporting, and other sources of bias) [18]. A judgement was made regarding the risk of bias for each of these domains using three categories "low," "high," and "unclear" if the study details were insufficient. Any disparity regarding risk of bias was discussed and resolved by consultation with the principal author.

2.5. Statistical Analysis. We performed a preliminary narrative synthesis of the data. Depending on the heterogeneity of the studies, we performed either a fixed-effect or a random effect meta-analysis. The heterogeneity of the trials was assessed through visual inspection of forest plots and the calculation of the $I^{2}$ statistic using a $50 \%$ limit to indicate substantial heterogeneity [19]. End point scores were expressed as standardized mean differences (SMDs) with associated 95\% confidence intervals (CIs). We performed the meta-analysis using RevMan 5 software [20]. The risk of publication bias was assessed graphically by a funnel plot [21], and the robustness of the overall estimates obtained was assessed by sensitivity analyses.

\section{Results}

3.1. Characteristics of Included Studies. A total of 33,995 references were initially identified from the computerized search. After removing 24,779 duplicates, the titles of the remaining 9223 articles were screened, and 1004 abstracts were retrieved for further scrutiny. A total of 705 studies were selected and retrieved for a full review, of which 688 were excluded. Finally, 22 studies met the criteria as described in the "Methods," which were included in the analysis [11, 22-42]. The flow chart of literature retrieval and screening is shown in Figure 1. Table 1 summarizes the main characteristics of the included studies. A total of 1346 study subjects were included in the meta-analysis.
3.2. Risk of Bias in Included Studies. See Figures S1 and S2 in the Supplementary Material for the risk-of-bias assessment for included studies. Twenty out of 22 studies (90\%) were RCTs, with a low risk of bias for allocation. However, 17 of these RCTs did not provide details about the allocation concealment; thus, this item was considered with an unclear risk of bias. Given the nature of intervention in these studies (physical exercise), blinding of participants was not possible. In addition, blinding of outcome assessment was performed in two studies, whereas the remaining 20 studies had an unclear risk of bias for this item. Therefore, the overall quality was medium.

\subsection{Meta-Analysis Results}

3.3.1. Studies of the Effect of Physical Exercises on Pulmonary Rehabilitation in Children with Asthma. Fifteen studies evaluated the effects of physical exercise on FVC\% pred in children with asthma. A pooled analysis of the heterogeneous data $\left(I^{2}=36 \% ; P<0.0001\right)$ included a total of 458 intervention and 421 control participants. As shown in Figure 2(a), exercise has a significant advantage in improving $\mathrm{FVC} \%$ pred in children with bronchial asthma compared with the control group, and the difference among the groups is statistically significant $(\mathrm{SMD}=0.27$ and $95 \%$ CI: 0.13 to 0.40 ). Eighteen studies evaluated the effects of physical exercise on FEV1\% pred in children with asthma. A pooled analysis of the heterogeneous data $\left(I^{2}=19 \%\right.$; $P>0.05)$ included a total of 540 intervention and 503 control participants. The results of Figure 2(b) showed that the significant effect of exercise on improving FEV1\% pred indexes in children with bronchial asthma cannot be determined compared with the control group, and no significant difference was observed between the two groups $(\mathrm{SMD}=0.11$ and $95 \% \mathrm{CI}:-0.01$ to 0.24$)$. Six studies evaluated the effects of physical exercise on FEV25-75\% pred in children with asthma. A pooled analysis of the heterogeneous data $\left(I^{2}=69 \%\right.$; $\left.P>0.05\right)$ included a total of 103 intervention and 99 control participants. As shown in Figure 2(c), the significant effect of exercise on improving FEV25-75\% pred indexes in children with bronchial asthma cannot be determined compared with the control group, and no significant difference was observed between the two groups $(\mathrm{MD}=5.07$ and $95 \% \mathrm{CI}$ : -3.53 to 13.67$)$. Eleven studies evaluated the effects of physical exercise on PEF\% pred in children with asthma. A pooled analysis of the heterogeneous data $\left(I^{2}=72 \% ; P<0.01\right)$ included a total of 411 intervention and 365 control participants. As shown in Figure 2(d), compared with the control group, exercise had significant advantages in improving PEF\% pred indexes in children with bronchial asthma, and the difference among groups was statistically significant $(\mathrm{MD}=4.53$ and $95 \% \mathrm{CI}$ : 1.27 to 7.80$)$.

3.3.2. Studies of the Effect of Physical Exercises on Exercise Capacity in Children with Asthma. Five studies evaluated the effects of physical exercise on 6 MWT in children with asthma. A pooled analysis of the heterogeneous data 


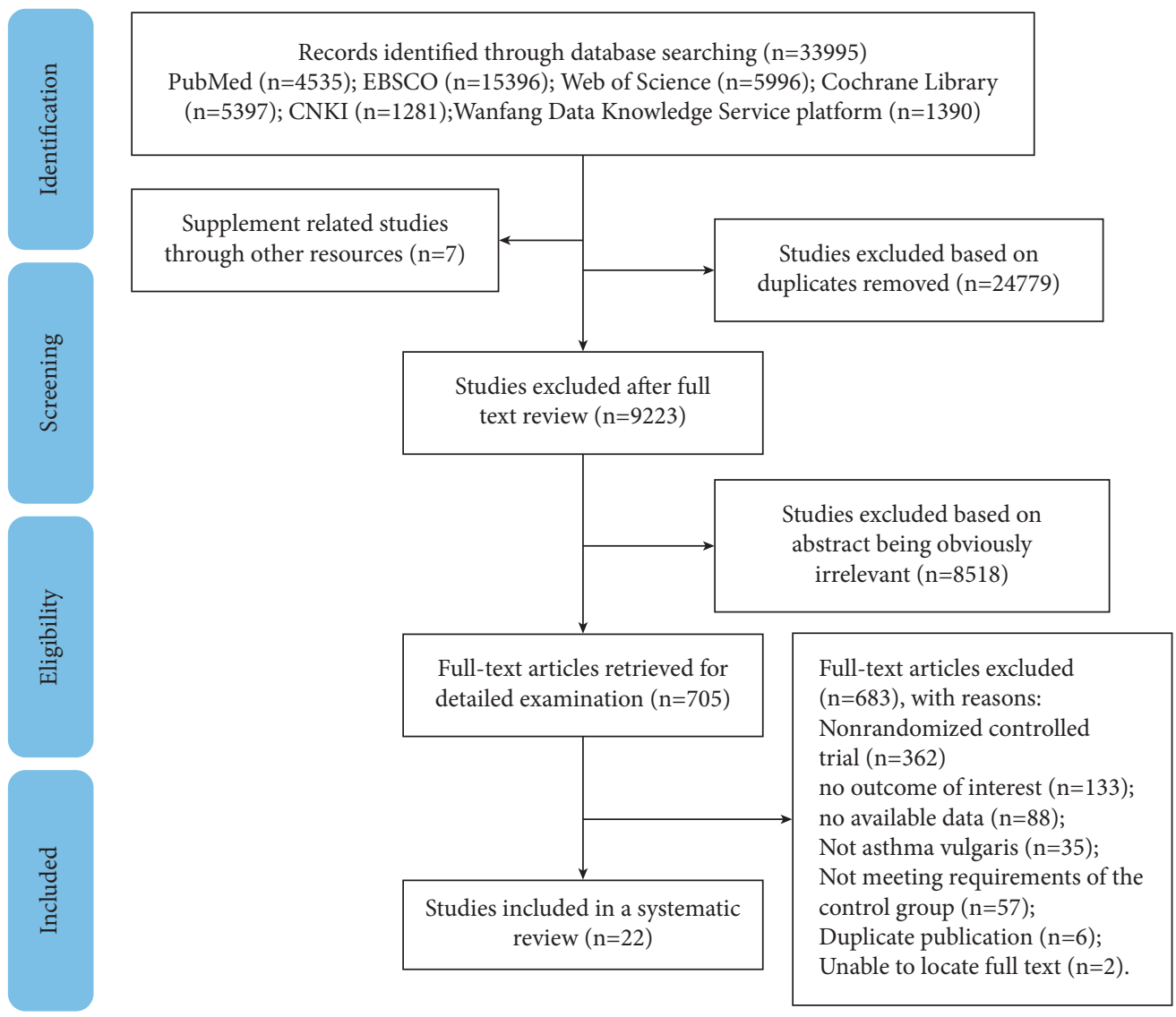

FIGURE 1: Literature screening process and results.

$\left(I^{2}=96 \% ; P<0.01\right)$ included a total of 132 intervention and 124 control participants. As shown in Supplementary Figure S3, exercise has significant advantages in improving the exercise ability of children with bronchial asthma compared with the control group, and the difference among the groups is statistically significant $(\mathrm{MD}=110.65$ and $95 \%$ CI: 31.95 to 189.34 ). The heterogeneity for $6 \mathrm{MWT}$ was reduced from $96 \%$ to $38 \%$ after the exclusion of the study by Latorre-Roma'n et al. [29].

Only three studies evaluated the effects of physical exercise on RPE in children with asthma. A pooled analysis of the heterogeneous data $\left(I^{2}=61 \% ; P<0.001\right)$ included a total of 92 intervention and 79 control participants. As shown in Supplementary Figure S4, exercise has significant advantages in improving the exercise ability of children with bronchial asthma compared with the control group, and the difference among the groups is statistically significant $(\mathrm{MD}=-2.28$ and 95\% CI: -3.21 to -1.36$)$. The heterogeneity for RPE was reduced from $61 \%$ to $0 \%$ after the exclusion of study by Latorre-Roma'n et al. [29].

Only three studies evaluated the effects of physical exercise on PP in children with asthma. A pooled analysis of the heterogeneous data $\left(I^{2}=69 \% ; P=0.001\right)$ included a total of 83 intervention and 82 control participants. As shown in Supplementary Figure S5, exercise has significant advantages in improving the exercise ability of children with bronchial asthma compared with the control group, and the difference among the groups is statistically significant $(\mathrm{MD}=0.94$ and 95\% CI: 0.37 to 1.52 ). The heterogeneity for PP was reduced from $69 \%$ to $0 \%$ after the exclusion of the study by Li et al. (2016) [31].

3.3.3. Studies of the Effect of Physical Exercises on Quality of Life in Children with Asthma. Ten studies evaluated the effects of physical exercise on quality of life in children with asthma. A pooled analysis of the heterogeneous data $\left(I^{2}=94 \% ; P<0.001\right)$ included a total of 363 intervention and 351 control participants. As shown in Figure 3, exercise has significant advantages in improving the quality of life of children with bronchial asthma compared with the control group, and the difference between the groups is statistically significant $(\mathrm{MD}=1.28$ and $95 \% \mathrm{CI}$ : 0.60 to 1.95$)$.

3.4. Subgroup Analysis. According to the subgroup analysis of different intervention times, the results showed that the exercise intervention of more than 8 weeks was significantly better than the routine control group in improving the indexes of FVC $\%$ pred, FEV1\% pred, FEV25-75\% pred, and PEF\% pred in children with bronchial asthma, and the difference was statistically significant (Table 2). 
TABLE 1: Characteristics of studies included.

\begin{tabular}{|c|c|c|c|c|c|c|c|}
\hline $\begin{array}{l}\text { First author. } \\
\text { (year).(reference) }\end{array}$ & $\begin{array}{l}\text { Sample size } \\
\text { (I/C) }\end{array}$ & $\begin{array}{c}\text { Mean age } \\
\left({ }^{*} \mathrm{I} / \mathrm{C}\right)\end{array}$ & Intervention & Control & $\begin{array}{c}\text { Intervention } \\
\text { duration }\end{array}$ & $\begin{array}{c}\text { Intervention } \\
\text { frequency }\end{array}$ & $\begin{array}{l}\text { Outcome } \\
\text { measure }\end{array}$ \\
\hline $\begin{array}{l}\text { Andrade et al. } \\
(2014) .[11]\end{array}$ & $27(10 / 17)$ & $\begin{array}{l}11.4 \pm 2.3 / \\
11.7 \pm 2.3\end{array}$ & Aerobic training & $\begin{array}{l}\text { Routine } \\
\text { activities }\end{array}$ & $6 \mathrm{w}$ & $\begin{array}{c}3 \text { times/week, } \\
20-30 \\
\text { minutes/time }\end{array}$ & $\begin{array}{c}\text { FVC\% pred, } \\
\text { FEV1\% pred, } \\
\text { PAQLQ, 6MWT }\end{array}$ \\
\hline $\begin{array}{l}\text { Asher et al. } \\
(1990) .[22]\end{array}$ & $38(19 / 19)$ & $\begin{array}{c}9.5 \pm 2.7 / \\
10 \pm 2.6\end{array}$ & $\begin{array}{l}\text { Physical therapy } \\
\text { based on exercise }\end{array}$ & $\begin{array}{c}\text { Routine } \\
\text { drug therapy }\end{array}$ & $2 \mathrm{w}$ & $\begin{array}{l}2 \text { times/day, } \\
20-30 \\
\text { minutes/time }\end{array}$ & $\begin{array}{l}\text { FVC\% pred, } \\
\text { FEV1\% pred, } \\
\text { FEF25-75\% } \\
\text { pred, PEF\% pred }\end{array}$ \\
\hline $\begin{array}{l}\text { Basaran et al. } \\
(2006) .[23]\end{array}$ & $58(30 / 28)$ & $\begin{array}{c}10.35 \pm 2.2 / \\
10.45 \pm 2.1\end{array}$ & $\begin{array}{c}\text { Submaximal aerobic } \\
\text { exercise }\end{array}$ & $\begin{array}{l}\text { Routine } \\
\text { activities }\end{array}$ & $8 \mathrm{w}$ & $\begin{array}{c}3 \text { times/week, } \\
55-60 \\
\text { minutes/time }\end{array}$ & $\begin{array}{l}\text { FVC\% pred, PEF } \\
\% \text { pred, PAQLQ, } \\
6 \mathrm{MWT}\end{array}$ \\
\hline $\begin{array}{l}\text { Karakoç et al. } \\
(2000) .[24]\end{array}$ & $28(16 / 12)$ & $\begin{array}{c}10.8 \pm 2.3 / \\
10.2 \pm 2.4\end{array}$ & $\begin{array}{l}\text { Lung rehabilitation } \\
\text { training program } \\
\text { based on exercise }\end{array}$ & $\begin{array}{l}\text { Routine } \\
\text { activities }\end{array}$ & $4 \mathrm{w}$ & - & $\begin{array}{l}\text { FVC } \% \text { pred, } \\
\text { FEV1\% pred, } \\
\text { FEF25-75\% } \\
\text { pred, PEF\% pred }\end{array}$ \\
\hline $\begin{array}{l}\text { Carew et al. } \\
\text { (2018). [25] }\end{array}$ & $37(27 / 10)$ & $\begin{array}{c}13.3 \pm 1.9 \\
13.2 \pm 1.6 \\
13.5 \pm 1.8 / \\
12.0 \pm 3.1\end{array}$ & $\begin{array}{l}\text { Swimming, football, } \\
\text { basketball }\end{array}$ & $\begin{array}{l}\text { Routine } \\
\text { activities }\end{array}$ & $6 \mathrm{w}$ & $\begin{array}{l}\text { Once a week, } \\
40 \text { minutes }\end{array}$ & $\begin{array}{l}\text { FVC\% pred, } \\
\text { FEV1\% pred, } \\
\text { PEF\% pred }\end{array}$ \\
\hline $\begin{array}{l}\text { Chen. } \\
\text { (2010). [26] }\end{array}$ & $99(50 / 49)$ & $\begin{array}{l}9.10 \pm 2.14 / \\
9.18 \pm 3.27\end{array}$ & Exercise prescription & $\begin{array}{c}\text { Routine } \\
\text { drug therapy }\end{array}$ & $16 \mathrm{w}$ & $\begin{array}{l}3 \text { times/week, } \\
20 \text { minutes/ } \\
\text { week }\end{array}$ & $\begin{array}{l}\text { FVC\% pred, } \\
\text { FEV1\% pred, } \\
\text { PEF\% pred, } \\
\text { PAQLQ }\end{array}$ \\
\hline $\begin{array}{l}\text { Chen et al. } \\
(2020) \text {. [27] }\end{array}$ & $40(20 / 20)$ & $\begin{array}{c}11.4 \pm 2.8 / \\
12.1 \pm 3.4\end{array}$ & $\begin{array}{l}\text { Fixed treadmill } \\
\text { exercise }\end{array}$ & $\begin{array}{l}\text { Routine } \\
\text { activities }\end{array}$ & $12 \mathrm{w}$ & $\begin{array}{l}3 \text { times/week, } \\
30 \text { minutes/ } \\
\text { week }\end{array}$ & $\begin{array}{l}\text { FVC\% pred, } \\
\text { FEV1\% pred, } \\
\text { PEF\% pred, } \\
6 \mathrm{MWT}, \mathrm{RPE}, \\
\text { PAQLQ }\end{array}$ \\
\hline $\begin{array}{l}\text { Counil et al. } \\
\text { (2003). [28] }\end{array}$ & $14(7 / 7)$ & $\begin{array}{c}14 \pm 0.6 / \\
13.9 \pm 0.8\end{array}$ & Power cycling & $\begin{array}{l}\text { Routine } \\
\text { activities }\end{array}$ & $6 \mathrm{w}$ & $\begin{array}{l}3 \text { times/week, } \\
45 \text { minutes/ } \\
\text { week }\end{array}$ & $\begin{array}{l}\text { FEV1\% pred, } \\
\text { FEF25-75\% } \\
\text { pred, PP }\end{array}$ \\
\hline $\begin{array}{l}\text { Latorre-Roma'n } \\
\text { et al. (2014). [29] }\end{array}$ & $105(58 / 47)$ & $\begin{array}{c}11.55 \pm 1.01 / \\
11.51 \pm 1.42\end{array}$ & $\begin{array}{l}\text { Indoor interval } \\
\text { training }\end{array}$ & $\begin{array}{l}\text { Routine } \\
\text { activities }\end{array}$ & $12 \mathrm{w}$ & $\begin{array}{l}3 \text { times/week, } \\
60 \text { minutes/ } \\
\text { week }\end{array}$ & $\begin{array}{c}\text { PAQLQ, } \\
\text { 6MWT, RPE }\end{array}$ \\
\hline $\begin{array}{l}\text { Leng et al. } \\
(2020) \text {. [30] }\end{array}$ & $60(30 / 30)$ & $\begin{array}{c}6.72 \pm 2.55 / \\
6.59 \pm 2.18\end{array}$ & Exercise prescription & $\begin{array}{c}\text { Routine } \\
\text { drug } \\
\text { treatment } \\
\text { and massage }\end{array}$ & $12 \mathrm{w}$ & $\begin{array}{l}5 \text { times/week, } \\
60 \text { minutes/ } \\
\text { week }\end{array}$ & $\begin{array}{l}\text { FVC } \% \text { pred, } \\
\text { FEV1\% pred }\end{array}$ \\
\hline $\begin{array}{l}\text { Li et al. } \\
(2016) .[31]\end{array}$ & $46(23 / 23)$ & $\begin{array}{c}11.9 \pm 2.3 / \\
12.5 \pm 3.0\end{array}$ & $\begin{array}{c}\text { Intermittent } \\
\text { anaerobic exercise } \\
\text { (pedaling) }\end{array}$ & $\begin{array}{l}\text { Routine } \\
\text { activities }\end{array}$ & $8 \mathrm{w}$ & $\begin{array}{l}3 \text { times/week, } \\
30 \text { seconds/ } \\
\text { time }\end{array}$ & $\begin{array}{l}\text { FVC\% pred, } \\
\text { FEV } 1 \% \text { pred, } \\
\text { PEF\% pred, PP }\end{array}$ \\
\hline $\begin{array}{l}\text { Li et al. } \\
(2016) .[32]\end{array}$ & $41(29 / 12)$ & $\begin{array}{l}11.7 \pm 2.0 \\
12.5 \pm 1.6 / \\
10.8 \pm 1.3\end{array}$ & $\begin{array}{l}\text { Medium intensity } \\
\text { continuous exercise; } \\
\text { high intensity } \\
\text { intermittent exercise }\end{array}$ & $\begin{array}{l}\text { Routine } \\
\text { activities }\end{array}$ & $8 \mathrm{w}$ & $\begin{array}{l}4 \text { times/week, } \\
40 \text { minutes/ } \\
\text { time or } \\
30 \text { seconds/ } \\
\text { time }\end{array}$ & $\begin{array}{l}\text { FVC\% pred, } \\
\text { FEV1\% pred, } \\
\text { PEF\% pred, } \\
6 \mathrm{MWT}, \mathrm{RPE}\end{array}$ \\
\hline $\begin{array}{l}\text { Li et al. } \\
\text { (2015). [33] }\end{array}$ & $128(64 / 64)$ & $8.8 \pm 2.4$ & Exercise prescription & $\begin{array}{c}\text { Routine } \\
\text { drug therapy }\end{array}$ & $24 \mathrm{w}$ & $\begin{array}{l}3 \text { times/week, } \\
20 \text { minutes/ } \\
\text { week }\end{array}$ & $\begin{array}{l}\text { FVC\% pred, } \\
\text { FEV1\% pred, } \\
\text { PEF\% pred, } \\
\text { PAQLQ }\end{array}$ \\
\hline $\begin{array}{l}\text { Moreira et al. } \\
(2008) .[34]\end{array}$ & $31(16 / 15)$ & $\begin{array}{c}12.9 \pm 3.4 / \\
12.5 \pm 3.5\end{array}$ & $\begin{array}{l}\text { Submaximal exercise } \\
\text { (aerobic exercise, } \\
\text { strength training, } \\
\text { balance and } \\
\text { coordination training, } \\
\text { relaxation exercise) }\end{array}$ & $\begin{array}{l}\text { Routine } \\
\text { activities }\end{array}$ & $12 \mathrm{w}$ & $\begin{array}{l}2 \text { times/week, } \\
50 \text { minutes/ } \\
\text { time }\end{array}$ & $\begin{array}{l}\text { FEV1\% pred, } \\
\text { FEF25-75\% } \\
\text { pred, PAQLQ }\end{array}$ \\
\hline $\begin{array}{l}\text { Onur et al. } \\
\text { (2011). [35] }\end{array}$ & $30(15 / 15)$ & $\begin{array}{l}9.8 \pm 1.8 / \\
10.3 \pm 2.0\end{array}$ & Cycling & $\begin{array}{c}\text { Routine } \\
\text { drug therapy }\end{array}$ & $8 \mathrm{w}$ & $\begin{array}{c}2 \text { times/week, } \\
60 \text { minutes/ } \\
\text { time }\end{array}$ & $\begin{array}{l}\text { FVC } \% \text { pred, } \\
\text { FEV1\% pred }\end{array}$ \\
\hline
\end{tabular}


TABle 1: Continued.

\begin{tabular}{|c|c|c|c|c|c|c|c|}
\hline $\begin{array}{l}\text { First author. } \\
\text { (year).(reference) }\end{array}$ & $\begin{array}{l}\text { Sample size } \\
\text { (I/C) }\end{array}$ & $\begin{array}{c}\text { Mean age } \\
\left({ }^{*} \mathrm{I} / \mathrm{C}\right) \\
\end{array}$ & Intervention & Control & $\begin{array}{c}\text { Intervention } \\
\text { duration }\end{array}$ & $\begin{array}{c}\text { Intervention } \\
\text { frequency }\end{array}$ & $\begin{array}{l}\text { Outcome } \\
\text { measure }\end{array}$ \\
\hline $\begin{array}{l}\text { Sanz-Santiago } \\
\text { et al. }(2020) .[36]\end{array}$ & $60(35 / 35)$ & $\begin{array}{l}12.1 \pm 2.1 / \\
11.1 \pm 2.9\end{array}$ & $\begin{array}{l}\text { Resistance and } \\
\text { aerobic training }\end{array}$ & $\begin{array}{l}\text { Routine } \\
\text { activities }\end{array}$ & $12 \mathrm{w}$ & $\begin{array}{l}3 \text { times/week, } \\
\text { 20-40 times/ } \\
\text { week, }\end{array}$ & PAQLQ \\
\hline Tan. (2019). [37] & $156(80 / 76)$ & $\begin{array}{l}8.24 \pm 2.13 / \\
8.08 \pm 2.17\end{array}$ & $\begin{array}{c}\text { Swimming, } \\
\text { intermittent sports } \\
\text { (table tennis, } \\
\text { badminton, } \\
\text { basketball, etc.) }\end{array}$ & $\begin{array}{c}\text { Routine } \\
\text { drug therapy }\end{array}$ & $12 \mathrm{w}$ & $\begin{array}{c}3 \text { times/week, } \\
20-30 \\
\text { minutes/time }\end{array}$ & $\begin{array}{l}\text { FVC\% pred, } \\
\text { FEV1\% pred, } \\
\text { PEF\% pred, } \\
\text { PAQLQ }\end{array}$ \\
\hline $\begin{array}{l}\text { Wang et al. } \\
(2009) .[38]\end{array}$ & $30(15 / 15)$ & $7-12$ & Swimming & $\begin{array}{l}\text { Routine } \\
\text { activities }\end{array}$ & $6 \mathrm{w}$ & $\begin{array}{l}3 \text { times/week, } \\
50 \text { minutes/ } \\
\text { week }\end{array}$ & $\begin{array}{l}\text { FVC\% pred, } \\
\text { FEV1\% pred, } \\
\text { FEF25-75\% } \\
\text { pred }\end{array}$ \\
\hline $\begin{array}{l}\text { Wicher et al. } \\
(2010) . \text { [39] }\end{array}$ & $61(30 / 31)$ & $\begin{array}{l}10.35 \pm 3.13 / \\
10.90 \pm 2.63\end{array}$ & Swimming & $\begin{array}{c}\text { Routine } \\
\text { drug therapy }\end{array}$ & $12 \mathrm{w}$ & $\begin{array}{l}2 \text { times/week, } \\
60 \text { minutes/ } \\
\text { time }\end{array}$ & $\begin{array}{l}\text { FVC\% pred, } \\
\text { FEV1\% pred, } \\
\text { FEF25-75\% } \\
\text { pred }\end{array}$ \\
\hline $\begin{array}{l}\text { Yang et al. } \\
(2020) .[40]\end{array}$ & $105(53 / 52)$ & $\begin{array}{l}8.24 \pm 1.27 / \\
8.68 \pm 1.36\end{array}$ & $\begin{array}{c}\text { Intermittent } \\
\text { anaerobic exercise } \\
\text { training }\end{array}$ & $\begin{array}{l}\text { Routine } \\
\text { breathing } \\
\text { training }\end{array}$ & $8 \mathrm{w}$ & $\begin{array}{l}7 \text { times/week, } \\
30 \text { minutes/ } \\
\text { time }\end{array}$ & $\begin{array}{l}\text { FEV1\% pred, } \\
\text { PEF\% pred, pp }\end{array}$ \\
\hline $\begin{array}{l}\text { Zhang et al. } \\
\text { (2019). [41] }\end{array}$ & $72(36 / 36)$ & $\begin{array}{c}6.9 \pm 2.3 / \\
7.1 \pm 2.7\end{array}$ & Aerobic training & $\begin{array}{c}\text { Routine } \\
\text { drug therapy }\end{array}$ & $6 \mathrm{w}$ & $\begin{array}{l}3 \text { times/week, } \\
40 \text { minutes/ } \\
\text { week }\end{array}$ & FEV1\% pred \\
\hline $\begin{array}{l}\text { Zhang et al. } \\
(2020) .[42]\end{array}$ & $80(40 / 40)$ & $\begin{array}{l}8.2 \pm 2.5 / \\
7.9 \pm 2.2\end{array}$ & $\begin{array}{l}\text { Aerobic exercise and } \\
\text { anaerobic exercise }\end{array}$ & $\begin{array}{l}\text { Routine } \\
\text { activities } \\
\text { and health } \\
\text { education }\end{array}$ & $8 \mathrm{w}$ & $\begin{array}{c}\text { 3-5 times/ } \\
\text { week, } \\
35-50 \\
\text { minutes/week }\end{array}$ & PAQLQ \\
\hline
\end{tabular}

I: intervention group; C: control group; w: week; FVC\% pred: pulmonary function indices include forced vital capacity to predicted value ratio; FEV1\% pred: forced expiratory volume at $1 \mathrm{~s}$ to predicted value ratio; FEF25-75\% pred: forced expiratory flow between $25 \%$ and $75 \%$ of vital capacity ratio; PEF\% pred: peak expiratory flow to predicted value ratio; PAQLQ: Pediatric Asthma Quality of Life Questionnaire; 6 MWT: the 6-minute walk test; RPE: rating of perceived effort; PP: peak power; —: no data.

\begin{tabular}{|c|c|c|c|c|c|c|c|c|c|c|c|c|}
\hline \multirow{3}{*}{$\begin{array}{l}\text { Study or Subgroup } \\
\text { Andrade } 2014\end{array}$} & \multicolumn{3}{|c|}{ Intervention } & \multicolumn{3}{|c|}{ control } & \multirow{3}{*}{$\begin{array}{c}\text { Weight } \\
(\%)\end{array}$} & \multirow{3}{*}{$\begin{array}{c}\text { Std. Mean Difference } \\
\text { IV, Fixed, 95\% CI } \\
0.97[0.14,1.80]\end{array}$} & \multirow{2}{*}{\multicolumn{4}{|c|}{$\begin{array}{l}\text { Std. Mean Difference } \\
\text { IV, Fixed, 95\% CI }\end{array}$}} \\
\hline & \multirow{2}{*}{$\begin{array}{c}\text { Mean } \\
2.7\end{array}$} & \multirow{2}{*}{$\begin{array}{l}\text { SD } \\
0.2\end{array}$} & \multirow{2}{*}{$\begin{array}{c}\text { Total } \\
10\end{array}$} & \multirow{2}{*}{$\frac{\text { Mean }}{2.5}$} & \multirow{2}{*}{$\begin{array}{c}\text { SD } \\
0.2\end{array}$} & \multirow{2}{*}{$\begin{array}{c}\text { Total } \\
17\end{array}$} & & & & & & \\
\hline & & & & & & & & & & & & \\
\hline Asher 1990 & 95 & 19 & 19 & 100 & 18 & 19 & 4.5 & $-0.26[-0.90,0.37]$ & & & & \\
\hline Basaran 2006 & 82.6 & 10.1 & 30 & 82.5 & 12.3 & 28 & 6.9 & $0.01[-0.51,0.52]$ & & & & \\
\hline Bingöl karakoç 2000 & 84.75 & 10.76 & 16 & 81.41 & 7.07 & 12 & 3.2 & $0.35[-0.41,1.10]$ & & & & \\
\hline Carew 2018 & 100.4 & 13.4 & 27 & 96.5 & 9.9 & 10 & 3.4 & $0.30[-0.43,1.03]$ & & & & \\
\hline Chen Boli 2010 & 96.84 & 12.84 & 50 & 93.45 & 14.62 & 49 & 11.7 & $0.24[-0.15,0.64]$ & & & & \\
\hline Chen Qiong 2020 & 81.4 & 9.5 & 20 & 79.3 & 6.2 & 20 & 4.7 & $0.26[-0.37,0.88]$ & & & & \\
\hline Leng Ling 2020 & 101.02 & 14.31 & 30 & 99.1 & 10.84 & 30 & 7.1 & $0.15[-0.36,0.66]$ & & & & \\
\hline Li Guangxin 2016 & 83.7 & 9.8 & 23 & 81.1 & 12 & 23 & 5.4 & $0.23[-0.35,0.81]$ & & & & \\
\hline Li Jia 2016 & 89.5 & 8.7 & 29 & 93.5 & 9.8 & 12 & 3.9 & $-0.43[-1.11,0.25]$ & & & - & \\
\hline Li Tao 2015 & 97.6 & 12.3 & 64 & 93.7 & 11.2 & 64 & 15.0 & $0.33[-0.02,0.68]$ & & & & \\
\hline Onur 2011 & 90.8 & 11.2 & 15 & 84.7 & 14.7 & 15 & 3.5 & $0.45[-0.27,1.18]$ & & & & \\
\hline Tan Jing 2019 & 96.49 & 3.89 & 80 & 94.64 & 8.77 & 76 & 18.3 & $0.27[-0.04,0.59]$ & & & & \\
\hline Wang 2009 & 101 & 16.252 & 15 & 101 & 23.475 & 15 & 3.6 & $0.00[-0.72,0.72]$ & & & & \\
\hline Wicher 2010 & 97.77 & 12.34 & 30 & 82.73 & 13.4 & 31 & 6.2 & $1.15[0.61,1.70]$ & & & & \\
\hline Total $(95 \%$ CI) & & & 458 & & & 421 & 100.0 & $0.27[0.13,0.40]$ & & & $>$ & \\
\hline Heterogeneity: $\mathrm{Chi}^{2}=$ & $21.81, \mathrm{~d} f$ & $=14(P=$ & $=0.08)$; & $I^{2}=36$ & & & & & & & & \\
\hline Test for overall effect & $Z=3.90$ & $(P<0.00$ & & & & & & & -1 & -0.5 & 0.5 & 1 \\
\hline & & & & & & & & & Favours (co & ntrol) & Favours & (Intervention) \\
\hline
\end{tabular}

(a)

FIgURE 2: Continued. 


\begin{tabular}{|c|c|c|c|c|c|c|c|c|c|c|c|c|}
\hline \multirow{2}{*}{ Study or Subgroup } & \multicolumn{3}{|c|}{ Intervention } & \multicolumn{3}{|c|}{ control } & \multirow{2}{*}{$\begin{array}{c}\text { Weight } \\
(\%)\end{array}$} & \multirow{2}{*}{$\begin{array}{l}\text { Std. Mean Difference } \\
\text { IV, Fixed, } 95 \% \text { CI }\end{array}$} & & \multirow{2}{*}{\multicolumn{2}{|c|}{$\begin{array}{l}\text { Std. Mean Difference } \\
\text { IV, Fixed, 95\% CI }\end{array}$}} & \\
\hline & Mean & SD & Total & Mean & SD & Total & & & & & & \\
\hline Andrade 2014 & 2.2 & 0.2 & 10 & 2.2 & 0.1 & 17 & 2.5 & $0.00[-0.78,0.78]$ & & & & \\
\hline Asher 1990 & 87 & 18 & 19 & 86 & 21 & 19 & 3.8 & $0.05[-0.59,0.69]$ & & & & \\
\hline Bingöl karakoç 2000 & 80.62 & 12.27 & 16 & 75.91 & 5.43 & 12 & 2.6 & $0.46[-0.30,1.22]$ & & & & \\
\hline Carew 2018 & 98.2 & 13.5 & 27 & 93.3 & 8.6 & 10 & 2.8 & $0.39[-0.35,1.12]$ & & & & \\
\hline Chen Boli 2010 & 107.99 & 15.79 & 50 & 103.98 & 17.35 & 49 & 9.7 & $0.24[-0.16,0.64]$ & & & & \\
\hline Chen Qiong 2020 & 85.4 & 10.3 & 20 & 84.1 & 9.9 & 20 & 3.9 & $0.13[-0.49,0.75]$ & & & & \\
\hline Council 2003 & 96.7 & 2.3 & 7 & 96.6 & 4.3 & 7 & 1.4 & $0.03[-1.02,1.07]$ & & & & \\
\hline Leng Ling 2020 & 96.84 & 13.55 & 30 & 100.87 & 9.93 & 30 & 5.8 & $-0.33[-0.84,0.17]$ & & & & \\
\hline Li Guangxin 2016 & 86.9 & 11.8 & 23 & 84.9 & 10.5 & 23 & 4.5 & $0.18[-0.40,0.76]$ & & & & \\
\hline Li Jia 2016 & 85.7 & 8.8 & 29 & 86.6 & 9.2 & 12 & 3.3 & $-0.10[-0.77,0.57]$ & & & & \\
\hline Li Tao 2015 & 109.7 & 14.7 & 64 & 103.2 & 13.8 & 64 & 12.3 & $0.45[0.10,0.80]$ & & & & \\
\hline Moreira 2008 & 87.7 & 8.8 & 16 & 90.13 & 10.3 & 15 & 3.0 & $-0.25[-0.96,0.46]$ & & & & \\
\hline Onur 2011 & 96 & 11.4 & 15 & 87.7 & 19.2 & 15 & 2.9 & $0.51[-0.22,1.24]$ & & & & \\
\hline Tan Jing 2019 & 105.09 & 7.93 & 80 & 103.52 & 6.85 & 76 & 15.3 & $0.21[-0.10,0.53]$ & & & & \\
\hline Wang 2009 & 107 & 18.1 & 15 & 105 & 19.9 & 15 & 3.0 & $0.10[-0.61,0.82]$ & & & & \\
\hline Wicher 2010 & 81.9 & 13.09 & 30 & 90.56 & 12.07 & 31 & 5.7 & $-0.68[-1.20,-0.16]$ & & & & \\
\hline Yang Ge 2020 & 86.98 & 11.97 & 53 & 84.87 & 10.64 & 52 & 10.3 & $0.18[-0.20,0.57]$ & & & & \\
\hline Zhang 2019 & 91.2 & 3.5 & 36 & 91.4 & 3.3 & 36 & 7.1 & $-0.06[-0.52,0.40]$ & & & & \\
\hline \multirow{3}{*}{\multicolumn{9}{|c|}{$\begin{array}{l}\text { Total }(95 \% \mathrm{CI}) \\
\text { Heterogeneity: } \mathrm{Chi}^{2}=21.07, \mathrm{~d} f=17(P=0.22) ; I^{2}=19 \% \\
\text { Test for overall effect: } Z=1.80(P=0.07)\end{array}$}} & & & & \\
\hline & & & & & & & & & & & & \\
\hline & & & & & & & & & -1 & -0.5 & 0.5 & 1 \\
\hline \multicolumn{9}{|c|}{ Test for overall effect: $Z=1.80(P=0.07)$} & Favours & ontrol) & Favours & ntervention) \\
\hline
\end{tabular}

(b)

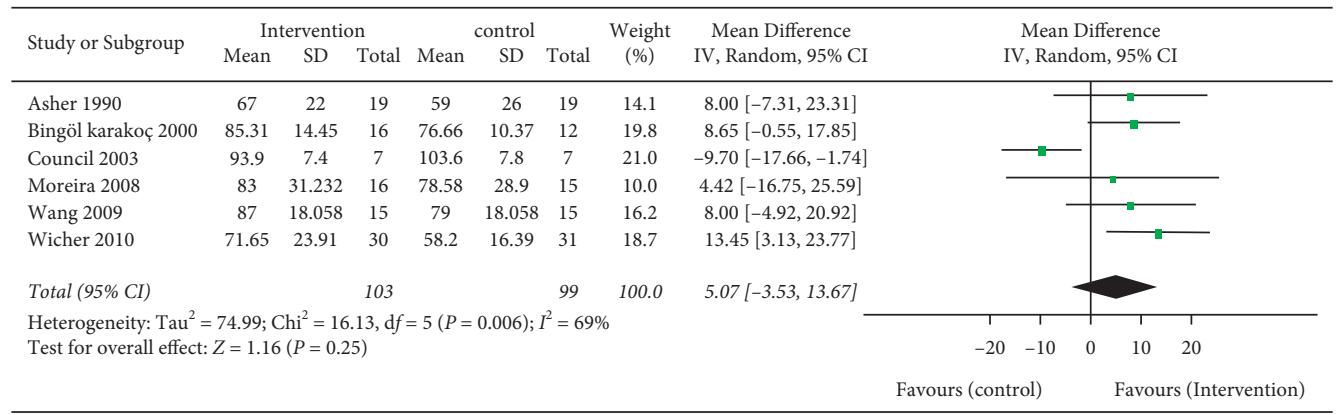

(c)

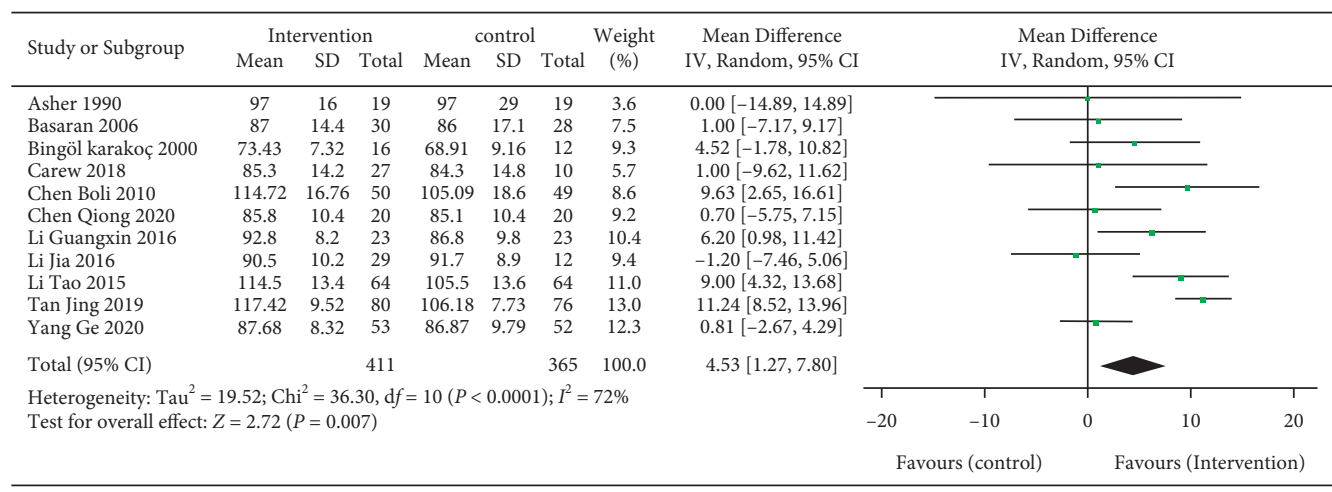

(d)

FIgURE 2: The effect of exercise on pulmonary rehabilitation. The effect of exercise on (a) FVC\%pred index; (b) FEV1\%pred index; (c) FEV25-75\%pred index; and (d) PEF\%pred index.

3.5. Sensitivity Analysis. According to the strict quality evaluation, the 22 articles included were selected to draw the funnel chart with the number of observation indexes $\geq 5$, such as FVC\% pred, FEV1\% pred, FEV25-75\% pred, PEF\% pred, 6MWT, and quality of life score (Figure 4). The asymmetry of the funnel chart can be seen from the chart, which may be related to the inconsistency of specific intervention methods, treatment frequency, and outcome measurement methods in the included RCTs; that is, real heterogeneity might be observed among studies, not necessarily publication bias.

\section{Discussion}

In recent years, the prevalence rate of bronchial asthma in children around the world has increased significantly, and the overall control level remains unsatisfactory [43]. If we cannot get timely and effective treatment, then the condition of some patients will continue into adulthood, which brings a huge economic burden to the family and a huge consumption of medical and health resources to the country [16]. Therefore, the prevention and treatment of bronchial asthma in children should be performed as early as possible 


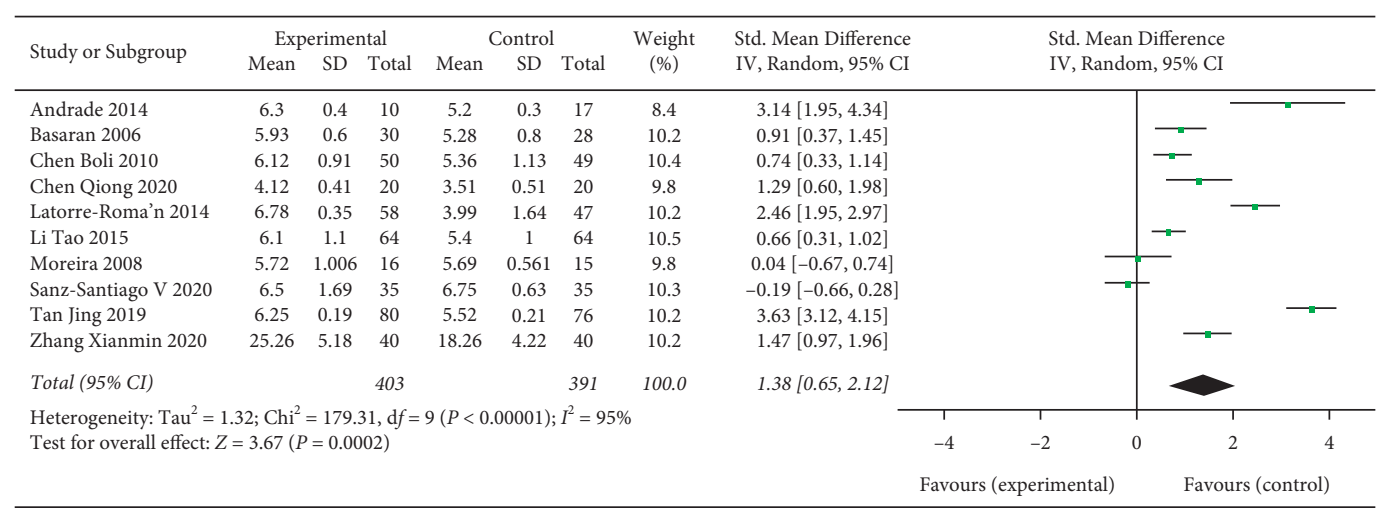

(a)

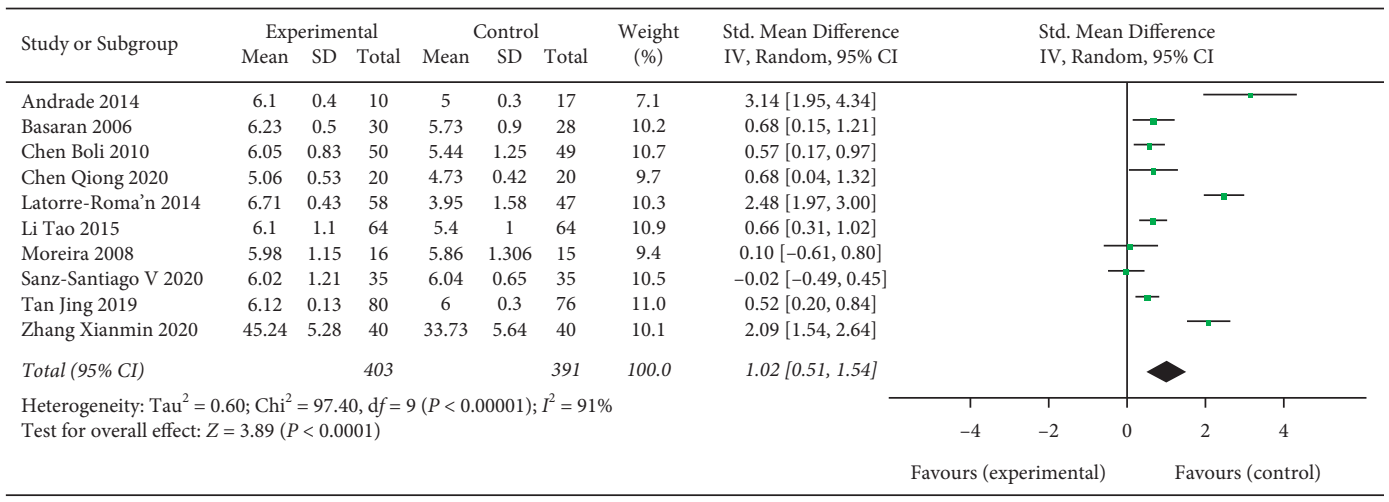

(b)

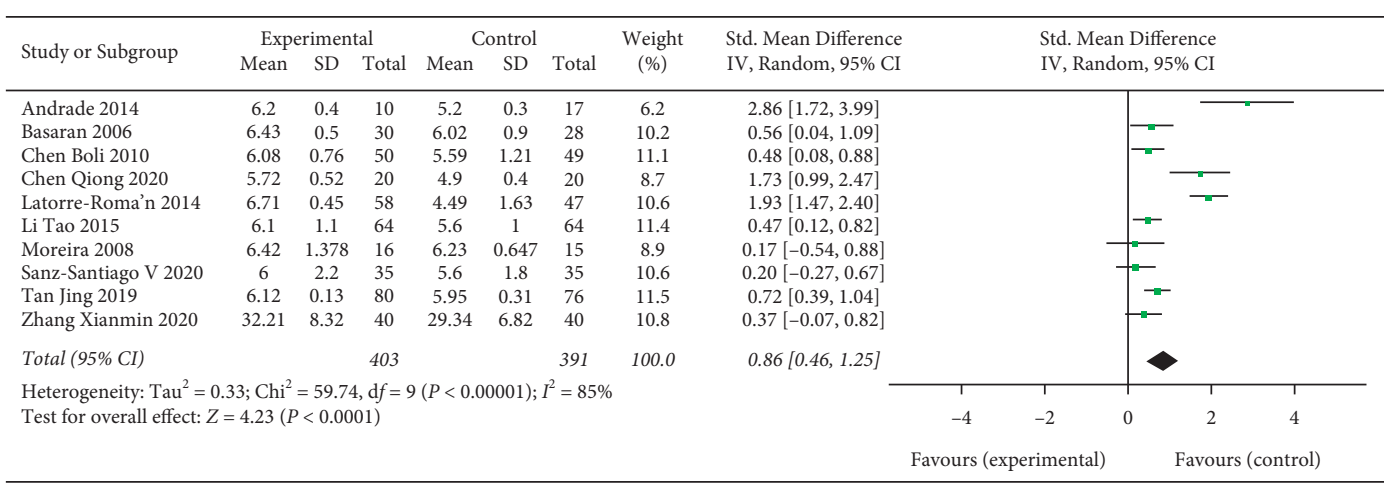

(c)

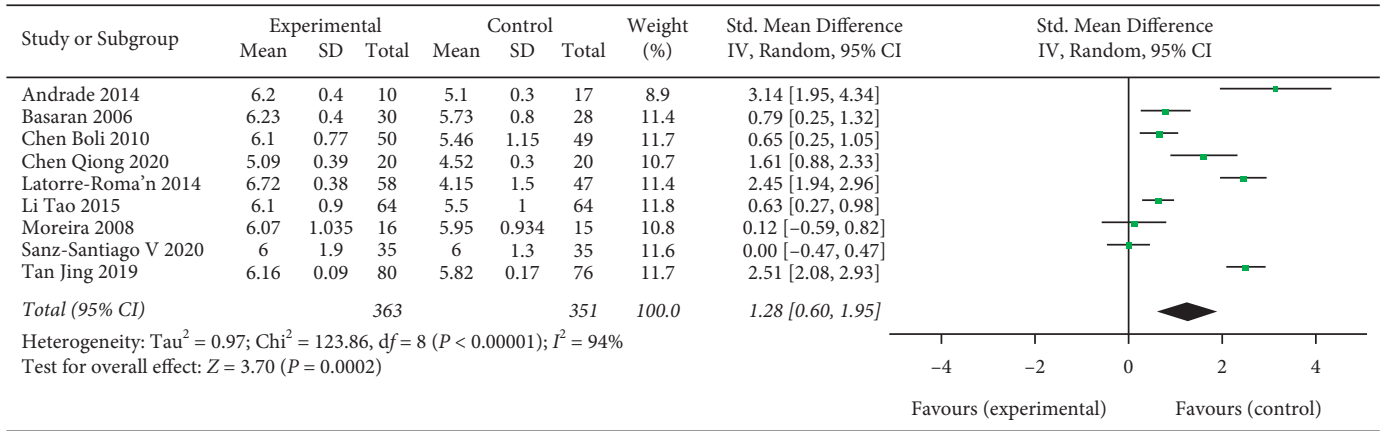

(d)

Figure 3: The effect of exercise on the quality of life. The effect of exercise on (a) activity score; (b) symptom score; (c) emotional score; and (d) total score. 
TABLE 2: Subgroup analysis results.

\begin{tabular}{|c|c|c|c|c|c|c|}
\hline \multirow[t]{2}{*}{ Subgroup (intervention time) } & \multirow[t]{2}{*}{ Studies } & \multicolumn{2}{|c|}{$\begin{array}{l}\text { Heterogeneity test } \\
\text { results }\end{array}$} & \multirow[t]{2}{*}{ Effect model } & \multicolumn{2}{|c|}{ Meta-analysis results } \\
\hline & & $P$ & $I^{2}(\%)$ & & MD/SMD (95\% CI) & Pvalue \\
\hline \multicolumn{7}{|l|}{ FVC\% pred } \\
\hline$\geq 8 \mathrm{w}$ & 10 & 0.01 & 56 & Fixed & $2.62(1.19,4.04)$ & 0.0003 \\
\hline$<8 \mathrm{w}$ & 5 & 0.21 & 32 & Fixed & $0.20(-0.12,0.53)$ & 0.22 \\
\hline \multicolumn{7}{|l|}{ FEV1\% pred } \\
\hline$\geq 8 \mathrm{w}$ & 11 & 0.02 & 52 & Fixed & $1.09(-0.38,2.56)$ & 0.15 \\
\hline$<8 \mathrm{w}$ & 7 & 0.92 & 0 & Fixed & $0.10(-0.15,0.36)$ & 0.42 \\
\hline \multicolumn{7}{|l|}{ FEV25-75\% pred } \\
\hline$\geq 8 \mathrm{w}$ & 2 & 0.45 & 0 & Fixed & $11.72(2.44,20.99)$ & 0.01 \\
\hline$<8 \mathrm{w}$ & 4 & 0.009 & 74 & Random & $3.02(-7.62,13.67)$ & 0.58 \\
\hline \multicolumn{7}{|l|}{ PEF\% pred } \\
\hline$\geq 8 \mathrm{w}$ & 8 & $<0.0001$ & 80 & Random & $4.94(1.07,8.82)$ & 0.01 \\
\hline$<8 \mathrm{w}$ & 3 & 0.77 & 0 & Fixed & $3.18(-1.91,8.28)$ & 0.22 \\
\hline
\end{tabular}

FVC\% pred: pulmonary function indices include forced vital capacity to predicted value ratio; FEV $1 \%$ pred: forced expiratory volume at $1 \mathrm{~s}$ to predicted value ratio; FEF $25-75 \%$ pred: forced expiratory flow between $25 \%$ and $75 \%$ of vital capacity ratio; PEF\% pred: peak expiratory flow to predicted value ratio; SMD: standardized mean difference; MD: mean difference.

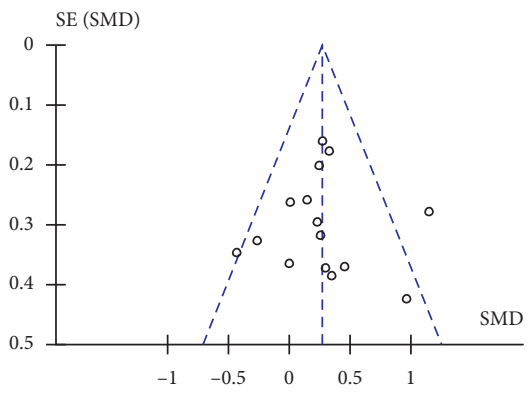

(a)

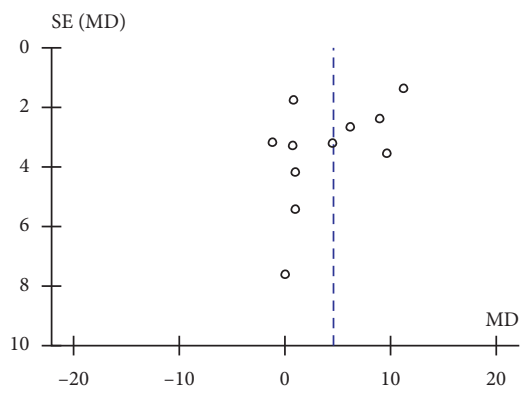

(d)

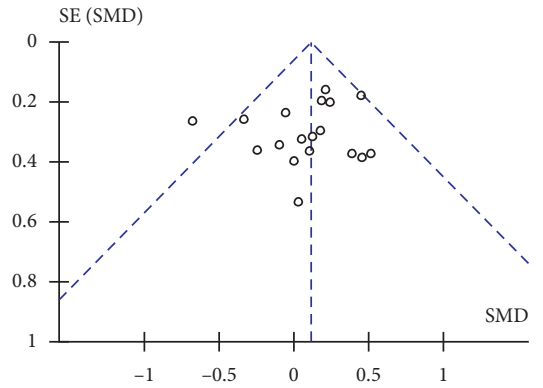

(b)

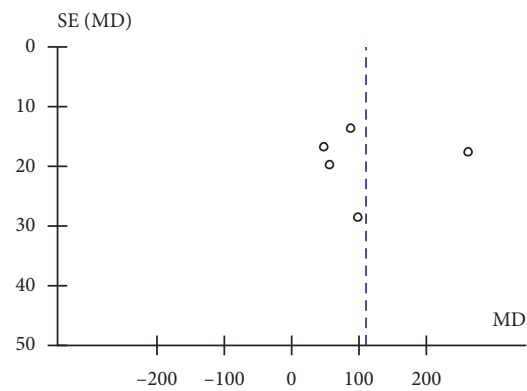

(e)

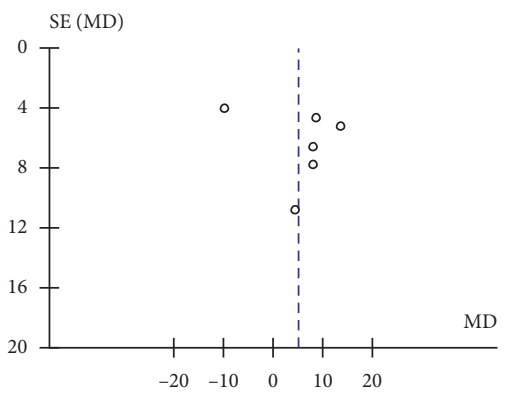

(c)

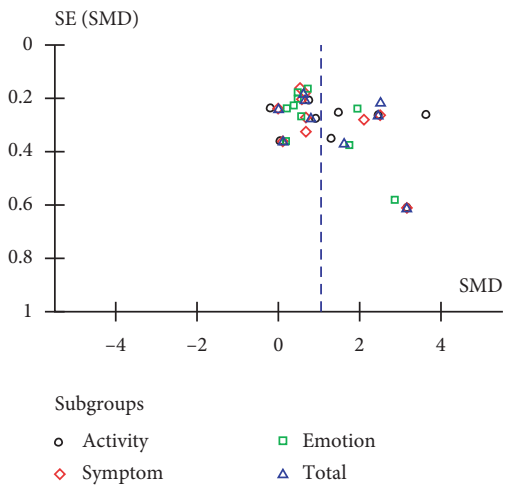

(f)

FIGURe 4: Funnel plot of the meta-analysis of the data. The funnel plot of (a) FVC\% pred; (b) FEV1\% pred; (c) FEV25-75\% pred; (d) PEF\% pred; (e) 6MWT; and (f) Quality of life scoring index.

and should adhere to the principles of long-term persistence, standardization, and personalized treatment [44]. Exercise improves healthy growth and development of children, but exercise in children with asthma remains a great concern because they have asthma-related symptoms in the course of exercise and they may avoid exercise because of fear. In addition, given the vague understanding of children with asthma participating in exercise, parents will not encourage or even restrict their children to participate in exercise.
Therefore, whether exercise can be used as a complementary alternative therapy for the rehabilitation of lung function in children with bronchial asthma and whether it can effectively improve the exercise ability and quality of life of children lacks relevant reports at home and abroad $[12,22,45]$. Further promoting the orderly application of exercise intervention in the field of rehabilitation of chronic respiratory diseases promotes not only the universal development of sports in the whole process of prevention, 
treatment, and rehabilitation of respiratory diseases, but also the high-quality development of medical and physical integration. In this study, through evidence-based medicine, the quantitative results show that exercise is significantly better than routine treatment in improving exercise ability, lung function, and quality of life of children with bronchial asthma, and the difference is statistically significant.

With the deepening of the study of exercise in children with bronchial asthma, the relevant research conclusions are still controversial. The results of this study show that the pulmonary function index, as an important adjustment index for the evaluation, treatment, and severity monitoring of bronchial asthma, has always been the focus of respiratory doctors [46]. In this study, we collected 22 items of RCT and evaluated the effectiveness of exercise and routine treatment in interfering with the recovery of pulmonary function in children with bronchial asthma. The results showed that exercise was significantly better than routine treatment in improving $\mathrm{FVC} \%$ pred, $\mathrm{PEF} \%$ pred, and other pulmonary function indexes in children with asthma $(p<0.01)$. However, no significant difference was observed in the improvement of FEV1\% pred and FEF25-75\% pred between the two groups $(p>0.05)$. Subgroup analysis further showed that the exercise intervention of more than 8 weeks was significantly better than routine treatment in improving the indexes of FVC\% pred, FEV1\% pred, FEV25-75\% pred, and PEF\% pred in children with bronchial asthma. Therefore, the exercise intervention time for children with asthma should be maintained at more than 8 weeks.

The decrease of physical fitness (such as muscle strength and endurance and cardiopulmonary function) caused by the decrease of physical activity in children with bronchial asthma is an important factor in the decline of their quality of life and control of the disease [47]. Therefore, improving exercise ability and improving quality of life play a positive role in alleviating asthma symptoms. The 6-minute walking test (6MWT) is a simple, safe, and well-tolerated submaximal exercise test for adults and children with chronic heart or respiratory diseases. It is an effective tool to reflect the exercise ability and disease severity of subjects by measuring their walking distance within $6 \mathrm{~min}$ [48]. In this study, compared with the control group, the 6MWT distance of the exercise group increased, and a significant difference was observed $(p<0.001)$. The fatigue degree (RPE) of the exercise group after exercise was lower than that of the control group $(p<0.001)$. Therefore, exercise can improve the exercise ability of children with asthma and increase their exercise endurance in daily life. In addition, the $30 \mathrm{~s}$ Wingate test with an anaerobic power bicycle was used to detect the exercise ability of children with asthma, and the obtained PP index could reflect the children's anaerobic exercise ability. In this study, the PP of the exercise group was significantly different from that of the control group $(p=0.001)$, suggesting that scientific and effective exercise training can improve the cardiopulmonary system oxygen transport capacity of children with asthma and benign adaptation of the body. Consequently, the exercise ability has been improved [49]. Philipp et al. [50] have also shown that exercise training can effectively relieve asthma symptoms and improve exercise ability.
The Pediatric Asthma Quality of Life Questionnaire is a scale to evaluate the quality of life of children with asthma from 7 to 17 years old. It has high credibility, and it can accurately reflect the changes of the disease [51]. In this study, the observed symptoms, limitations of activity, emotional function, and total score of the exercise group were significantly different from those of the control group $(p<0.001)$, and the score was significantly higher than that of the control group, indicating that scientific and reasonable exercise training can improve the quality of life of children with asthma. This result is similar to that of the study by Fanelli et al. [52] on 38 children with moderate and severe persistent asthma for 16 weeks and Basaran et al. [23] on 62 children with mild-to-moderate asthma for 8 weeks. The results are the same as the results of the studies of aerobic training for 16 weeks in 38 children with moderate and severe persistent asthma and 8 weeks of moderate basketball training in 62 children with mild and moderate asthma.

The inclusion of literature in this meta-analysis still has limitations. First, a small number of included articles and research objects are identified, and some problems are found in the random grouping and blind implementation of some RCTs, which makes the research easy to be disturbed by subjective factors and affects the authenticity and reliability of the results. Second, in the intervention programs included in the study, the duration and frequency are not consistent, and the course of disease at the beginning of the intervention is also different, which has become an important factor affecting the curative effect to a certain extent. In addition, differences are observed in the rating scales and measurement methods used to measure outcome indicators, which leads to a decrease in the credibility of the results of the meta-analysis.

\section{Conclusion}

This meta-analysis of 22 randomized controlled trials shows that reasonable and effective exercise can significantly improve lung function, exercise ability, and quality of life in children with bronchial asthma. It also confirmed the effectiveness of exercise in these pulmonary function, exercise ability, and quality of life scores, which can be used as a reference basis for clinical exercise rehabilitation of children with asthma. However, considering the great differences in the specific treatment mode, the time, frequency, and course of disease at the beginning of intervention among different studies are limited by the quality and quantity of the included studies, and the specific clinical effects are still controversial. Therefore, conducting high-quality, large sample size RCTs is necessary for verification and evaluation in the future.

\section{Data Availability}

The data used to support the findings of this study are included within the supplementary information file.

\section{Conflicts of Interest}

The authors declare that there are no conflicts of interest regarding the publication of this paper. 


\section{Supplementary Materials}

Figure S1: risk assessment of bias in RCT. Figure S2: overall risk assessment of bias in RCT. Figure S3: the effect of exercise on 6MWT. Figure S4: the effect of exercise on RPE. Figure S5: the effect of exercise on PP. (Supplementary Materials)

\section{References}

[1] X. Z. Zhang, The Quality of Life and Related Factors Among Children with Asthma, Beijing University of Chinese Medicine, Beijing, China, 2017.

[2] A. J. Yang, "Guidelines for the diagnosis, prevention and treatment of bronchial asthma in children (2016 edition)," Chinese Medical Journal, vol. 53, no. 3, pp. 253-257, 2018.

[3] Y. Y. Li and B. H. Shi, "Research progress of exercise therapy in children with bronchial asthma," Modern Journal of Integrated Traditional Chinese and Western Medicine, vol. 26, no. 23, pp. 2620-2622, 2017.

[4] X. W. Chen, L. Q. Zhang, and H. Y. Zhou, "The effects of interval anaerobic training on the respiratory function and motor ability of asthma children with normal weight and overweight," Chinese Journal of Medical Frontiers, vol. 10, no. 2, pp. 56-59, 2018.

[5] L. Xiang, "Guide and analysis of the main points of the global initiative for asthma (GINA) 2018 update," Chinese Journal of Practical Pediatrics, vol. 33, no. 11, pp. 807-811, 2018.

[6] T. Qiu, S. Liang, and M. Dabbous, "COVID-19's Rehabilitation Guide of Integrated traditional Chinese and Western Medicine during his convalescent period (first Edition)," Tianjin Journal of Traditional Chinese Medicine, vol. 37, no. 5, pp. 484-489, 2020.

[7] L. S. Meng, E. D. Qiao, and Y. J. Li, "Meta-analysis of the effects of different Types of exercise on pulmonary function and quality of fife in COPD patients," Chinese Space Science Technology, vol. 56, no. 5, pp. 21-34, 2020.

[8] V. H. Wanrooij, M. Willeboordse, E. Dompeling, and K. D. van de Kant, "Exercise training in children with asthma: a systematic review," British Journal of Sports Medicine, vol. 48, no. 13, pp. 1024-1031, 2014.

[9] Z. G. Zheng, J. Y. Hu, and N. Liu, "Progress in the research of respiratory rehabilitation therapy 2017," Chinese Journal of practical Internal Medicine, vol. 38, no. 5, pp. 393-396, 2018.

[10] A. L. Kruis, N. Smidt, W. J. Assendelft et al., "Integrated disease management interventions for patients with chronic obstructive pulmonary disease," Cochrane Database of Systematic Reviews, vol. 10, no. 10, Article ID 009437, 2013.

[11] L. B. d. Andrade, M. C. A. Britto, N. Lucena-silva, R. G. Gomes, and J. N. Figueroa, "The efficacy of aerobic training in improving the inflammatory component of asthmatic children. Randomized trial," Respiratory Medicine, vol. 108, no. 10, pp. 1438-1445, 2014.

[12] S. Beggs, Y. C. Foong, H. C. Le, W. D. Mohammed Noor, J. A. E. Walters, and R. Wood-Baker, "Swimming training for asthma in children and adolescents aged 18 years and under," Cochrane Database of Systematic Reviews, vol. 4, Article ID 009607, 2013.

[13] W. Zhang, Q. Wang, L. Liu, W. Yang, and H. Liu, "Effects of physical therapy on lung function in children with asthma: a systematic review and meta-analysis," Pediatric Research, vol. 89, no. 6, pp. 1343-1351, 2021.

[14] J. G. Higgins and S. Green, Cochrane Handbook for Systematic Review Interventions, Cochrane, London, UK, 2017.
[15] D. Moher, A. Liberati, J. Tetzlaff, D. G. Altman, and PRISMA Group, "Preferred reporting items for systematic reviews and meta-analyses: the PRISMA statement," PLoS Medicine, vol. 6, no. 7, Article ID e1000097, 2009.

[16] Respiratory Group of Pediatrics Branch of Chinese Medical Association, "Guidelines for the diagnosis and prevention of bronchial asthma in children (2016 edition)," Chinese Journal of Pediatrics, vol. 54, no. 3, pp. 167-181, 2016.

[17] M. Masoli, D. Fabian, S. Holt, and R. Beasley, "The global burden of asthma: executive summary of the GINA Dissemination Committee Report," Allergy, vol. 59, no. 5, pp. 469-478, 2004.

[18] J. G. Higgins and S. Green, Cochrane Handbook for Systematic Review Interventions, Cochrane, London, UK, 2011.

[19] J. A. C. Sterne and M. Egger, "Funnel plots for detecting bias in meta-analysis," Journal of Clinical Epidemiology, vol. 54, no. 10, pp. 1046-1055, 2001.

[20] J. P. T. Higgins, S. G. Thompson, J. J. Deeks, and D. G. Altman, "Measuring inconsistency in meta-analyses," BMJ, vol. 327, no. 7414, pp. 557-560, 2003.

[21] Cochrane, Review Manager (RevMan) [Computer Program]. Version 5.3, The Nordic Cochrane Centre," The Cochrane Collaboration, Copenhagen, Denmark, 2014.

[22] M. I. Asher, C. Douglas, M. Airy, D. Andrews, and A Trenholme, "Effects of chest physical therapy on lung function in children recovering from acute severe asthma," Pediatric Pulmonology, vol. 9, no. 3, pp. 146-151, 1990.

[23] S. Basaran, F. Guler-uysal, N. Ergen, G. Seydaoglu, G. BingolKarakoç, and D. Ufuk Altintas, "Effects of physical exercise on quality of life, exercise capacity and pulmonary function in children with asthma," Journal of Rehabilitation Medicine, vol. 38, no. 2, pp. 130-135, 2006.

[24] G. Bingöl Karakoç, M. Yilmaz, S. Sur, D. Ufuk Altintas, T. Sarpel, and S. Güneter Kendirli, "The effects of daily pulmonary rehabilitation program at home on childhood asthma," Allergologia et Immunopathologia, vol. 28, no. 1, pp. 12-14, 2000.

[25] C. Carew and D. W. Cox, "Laps or lengths? The effects of different exercise programs on asthma control in children," Journal of Asthma, vol. 55, no. 8, pp. 877-881, 2018.

[26] B. L. Chen, Research on the Role of Exercise in the Prevention and Management of Childhood Asthma, Chongqing Medical University, Chongqing, China, 2010.

[27] Q. Chen, F. Q. Wang, and X. Sun, "Experimental study of aerobic exercise on improving the exercise capacity and quality of life of children with asthma," Journal of Guangzhou Institute of Physical Education, vol. 40, no. 5, pp. 125-128, 2020.

[28] F.-P. Counil, A. Varray, S. Matecki et al., "Training of aerobic and anaerobic fitness in children with asthma," The Journal of Pediatrics, vol. 142, no. 2, pp. 179-184, 2003.

[29] P. Á. Latorre-román, A. V. Navarro-martínez, and F. Garcíapinillos, "The effectiveness of an indoor intermittent training program for improving lung function, physical capacity, body composition and quality of life in children with asthma," Journal of Asthma, vol. 51, no. 5, pp. 544-551, 2014.

[30] L. Leng and L. Chi, "Observation on the effect of massage combined with exercise in the treatment of children with biased constitution in the remission period," Chinese Medical Innovation, vol. 17, no. 35, pp. 31-36, 2020.

[31] G. X. Li, L. Z. Ren, C. H. Yu, and L. Zhao, "Improvement effect of interval training on respiratory functionand exercise performance of asthmatic children," Journal of Jilin University(Medicine Edition), vol. 42, no. 05, pp. 949-953, 2016. 
[32] J. Li, X. Q. Wang, J. Lan, and J. Zhao, "Effects of high- intensity interval training and moderate- intensity continuous training on management of asthmatic children," Journal of Beijing Sport University, vol. 39, no. 10, pp. 58-64, 2016.

[33] T. Li, L. H. Li, and X. M. Bai, "Clinical study of montelukast sodium combined with exercise prescription on prevention and treatment in children with asthma," Journal of Clinical Pulmonary Medicine, vol. 20, no. 6, pp. 1099-1101, 2015.

[34] A. Moreira, L. Delgado, T. Haahtela et al., "Physical training does not increase allergic inflammation in asthmatic children," European Respiratory Journal, vol. 32, no. 6, pp. 1570-1575, 2008.

[35] E. Onur, C. Kabaroğlu, Ö. Günay et al., "The beneficial effects of physical exercise on antioxidant status in asthmatic children," Allergologia et Immunopathologia, vol. 39, no. 2, pp. 90-95, 2011.

[36] V. Sanz-Santiago, I. Diez-Vega, E. Santana-Sosa et al., "Effect of a combined exercise program on physical fitness, lung function, and quality of life in patients with controlled asthma and exercise symptoms: a randomized controlled trial," $P e$ diatric Pulmonology, vol. 55, no. 7, pp. 1608-1616, 2020.

[37] J. Tan, C. J. Liu, J. M. Huo et al., "A pplication of exercise prescriptions in the control of asthma," Chinese Journal of Child Health Care, vol. 27, no. 12, pp. 1331-1334, 2019.

[38] J.-S. Wang and W.-P. Hung, "The effects of a swimming intervention for children with asthma," Respirology, vol. 14, no. 6, pp. 838-842, 2009.

[39] I. B. Wicher, M. Â. G. D. O. Ribeiro, D. B. Marmo et al., "Avaliação espirométrica e da hiper-responsividade brônquica de crianças e adolescentes com asma atópica persistente moderada submetidos a natação," Jornal de Pediatria, vol. 86, no. 5, pp. 384-390, 2010.

[40] G. Yang, Y. Yang, and L. M. Mao, "The effect of 8-week interval anaerobic exercise training on children with mild asthma," Practical Clinical Medicine, vol. 21, no. 10, pp. 45-47, 2020.

[41] Y. F. Zhang and L. D. Yang, "Exercise training as an adjunctive therapy to montelukast in children with mild asthma: a randomized controlled trial," Medicine (Baltimore), vol. 98, no. 2, Article ID e14046, 2020.

[42] X. M. Zhang and Z. H. Wu, "Observation on the effect of exercise rehabilitation in children with bronchial asthma," Jilin Medicine, vol. 41, no. 08, pp. 2027-2030, 2020.

[43] National Pediatric Asthma Collaborative Group, "The third epidemiological survey of childhood asthma in Chinese cities," Chinese Journal of Pediatrics, vol. 51, no. 10, pp. 729-736, 2013.

[44] H. X. Yu, "Effect of Rehabilitation exercise prescription on Respiratory function of Adolescent patients with bronchial Asthma," Literature Review, vol. 27, no. 11, pp. 46-47, 2018.

[45] C. O. Francisco, S. A. Bhatawadekar, J. Babineau, W. D Reid, and A Yadollahi, "Effects of physical exercise training on nocturnal symptoms in asthma: Systematic review," PLoS One, vol. 13, no. 10, Article ID e0204953, 2018.

[46] Y. X. Wang, Study on HRCT and Lung Function Indexes of Chronic Obstructive Pulmonary Disease, Peking Union Medical College, Beijing, China, 2010.

[47] M. A. Mcnarry, L. M. Boddy, and G. S. Stratton, “The relationship between body mass index, aerobic performance and asthma in a pre-pubertal, population-level cohort," European Journal of Applied Physiology, vol. 114, no. 2, pp. 243-249, 2014.

[48] A. E. Lammers, A. A. Hislop, Y. Flynn, and S. G. Haworth, "The 6-minute walk test: normal values for children of 4-11 years of age," Archives of Disease in Childhood, vol. 93, no. 6, pp. 464-468, 2008.

[49] Y. M. Li, "Effect of high-intensity interval trainingon different training populations," Sports science, vol. 35, no. 8, pp. 59-75+96, 2015.

[50] A. Philipp, S. N. Diener, R. Kofmehl, and C. M. Spengler, "Effects of exercise training on airway hyperreactivity in asthma: a systematic review and meta-analysis," Sports Medicine, vol. 43, no. 11, pp. 1157-1170, 2013.

[51] J. Z. Wu, J. M. Zhang, L. L. Xu et al., “Application of pediatric asthma quality of life questionnaire to evaluate inhalation therapy in children with asthma," Chinese Journal of Pediatrics, vol. 4, pp. 63-64, 2004.

[52] A. Fanelli, A. L. B. Cabral, J. A. Neder, M. A. Martins, and C. R. F. Carvalho, "Exercise training on disease control and quality of life in asthmatic children," Medicine \& Science in Sports \& Exercise, vol. 39, no. 9, pp. 1474-1480, 2007. 